\title{
Operational Measure of Entanglement for Pure States of Bipartite Systems
}

\author{
Alexander A. Klyachko, Barış Öztop and Alexander S. Shumovsky ${ }^{\circledR}$ \\ Faculty of Science, Bilkent University, Bilkent, Ankara, 06800 Turkey \\ @ Corresponding author; E-mail: shumo@fen.bilkent.edu.tr
}

Received 1 August 2006

\begin{abstract}
We show that the complete information about the amount of entanglement carried by a bipartite pure state of any dimension (qubits, qutrits, etc), is determined by a local quantity that can be measured for either party of the system. This quantity is expressed in terms of averages of certain basic observables. In particular case of two entangled photon beams, this corresponds to the measurement of three Stokes parameters for either beam.
\end{abstract}

Keywords: entanglement, concurrence, local measure

PACS: 03.65.Ud, 03.67.Mn, 03.67.-a

The recent success in practical realization of quantum key distribution (see [1-3] and references therein) has been achieved through the use of specific quantum correlations between the parties in two-qubit systems. These correlations are peculiar to entangled states.

Further development of practical implementations of quantum information technologies requires simple physical measure of the amount of specific entanglement information carried by a given bipartite state. For two qubits, this quantity is expressed in terms of concurrence $C$ [4], which is widely used as a measure of entanglement.

The two representations of concurrence valid for pure states, that have been obtained in Refs. [4,5], express $C(\psi)$ in terms of complex coefficients of the bipartite state $\psi$ that hardly can be measured.

The aim of this note is to show that the amount of entanglement contained in a bipartite pure state $\psi$ can be measured locally in terms of the length of a certain vector with components given by averages of local basic observables. We prove universality of this measure for bipartite pure states of an arbitrary dimension. 
We consider a symmetric bipartite system with states defined in the Hilbert space $\mathcal{H}_{A} \otimes \mathcal{H}_{B}$, such that

$$
\operatorname{dim} \mathcal{H}_{A}=\operatorname{dim} \mathcal{H}_{B}=d=2,3, \cdots .
$$

A normalized pure state of such a system has the following form

$$
|\psi\rangle=\sum_{\ell, \ell^{\prime}=0}^{d-1} \psi_{\ell \ell^{\prime}}|\ell\rangle_{A} \otimes\left|\ell^{\prime}\right\rangle_{B}, \quad \sum_{\ell, \ell^{\prime}=0}^{d-1}\left|\psi_{\ell \ell^{\prime}}\right|^{2}=1 .
$$

According to Ref. [5], the corresponding concurrence is

$$
C(\psi)=\sqrt{\nu\left[1-\operatorname{Tr}\left(\rho_{r}^{2}\right)\right]}, \quad \nu=\frac{d}{d-1},
$$

where $\rho_{r}$ denotes the reduced density matrix (see also Ref. [6]).

It is has been known since pioneering works by Bell [7] that the quantum entanglement manifests itself by means of local measurements of certain observables performed at both parties of the system.

Generally speaking, definition of a quantum mechanical system assumes specification of both the Hilbert space of states and the basic observables that are allowed for obtaining information about the states [8]. Basic observables $X_{i}$ are found by examining the dynamic symmetry of the quantum system under consideration. Namely, they form an orthogonal basis of the Lie algebra $\mathcal{L}$ such that the corresponding Lie group is $[9,10]$

$$
G=\exp (i \mathcal{L})
$$

For example, a qubit is specified by states in the two-dimensional Hilbert space with the symmetry given by the dynamical group $G=S U(2)$ and basic observables provided by the three spin operators $S_{x}, S_{y}, S_{z}$, forming a basis of the $\mathcal{L}=s u(2)$ algebra. A qutrit is defined by states in the three-dimensional Hilbert space with dynamical symmetry $S U(3)$ and basic observables given by eight Gell-Mann matrices (orthogonal basis of the $s u(3)$ algebra) [11], and so on.

Below in this note we show that the amount of entanglement carried by the state (1) can be measured by means of the squared length of the vector

$$
\vec{R}(\psi)=\sum_{i}\left\langle\psi\left|X_{i}\right| \psi\right\rangle X_{i}
$$

in the Lie algebra $\mathcal{L}$, in other words, by means of the local quadratic form

$$
R^{2}(\psi)=\langle\psi|\vec{R}(\psi)| \psi\rangle=\sum_{i}\left[\operatorname{Tr}\left(\rho X_{i}\right)\right]^{2}, \quad \rho=|\psi\rangle\langle\psi|
$$

To clarify physical meaning of this measure, consider first the case of two qubits $(d=2)$, when (4) can be associated with the squared length of average spin vector

$$
R^{2}(\psi)=\left\langle\psi\left|S_{x}\right| \psi\right\rangle^{2}+\left\langle\psi\left|S_{y}\right| \psi\right\rangle^{2}+\left\langle\psi\left|S_{z}\right| \psi\right\rangle^{2}
$$


belonging to either party of the system. An indisputable advantage of the measure (5) is that it is directly expressed in terms of averages of observables of only one party rather than via the complex coefficients of bipartite state $\psi$ like in the case of conventional representation of concurrence for two-qubit pure state [4]

$$
C(\psi)=2|\operatorname{det}[\psi]|=2\left|\psi_{00} \psi_{11}-\psi_{01} \psi_{10}\right| \text {, }
$$

where $[\psi]$ is the $(2 \times 2)$ matrix of complex coefficients in the right-hand side of Eq. (1) with $d=2$ and $\ell=0,1$.

Note that despite the local character of the reduced density matrix $\rho_{r}$, the representation of concurrence (2) again expresses $C(\psi)$ in terms of complex coefficients $\psi_{\ell \ell^{\prime}}$ in (1).

In Ref. [12], we have proposed another representation of concurrence (2) in terms of the total uncertainty of measurement of basic observables (spin operators) for both parties

$$
C(\psi)=\sqrt{\frac{\mathbb{V}(\psi)-\mathbb{V}_{\min }}{\mathbb{V}_{\max }-\mathbb{V}_{\min }}} .
$$

Here

$$
\mathbb{V}(\psi)=\sum_{i} \sum_{J=A, B}\left(\left\langle\psi\left|\left(X_{i}^{J}\right)^{2}\right| \psi\right\rangle-\left\langle\psi\left|X_{i}^{J}\right| \psi\right\rangle^{2}\right)
$$

is the total variance (uncertainty) peculiar to the state $\psi$. Its maximal value corresponds to the completely entangled (CE) states, while the minimal value corresponds to unentangled (separable) states [10].

In Ref. [12], equivalence of representations (2) and (7) has been shown in the case of two qubits. We first show that the concurrence (7) can be expressed in terms of the measure (4) in either party of the system and hence has the local nature. Then, in Appendix, we prove equivalence of measures (2) and (7) for the state (1) with an arbitrary $d$.

Note first that in the case of symmetric bipartite systems under consideration, the form (4) for a given state $\psi$ has the same value for both parties. For the algebras of observables $s u(d)$ under consideration, there is the Casimir operator (c-number)

$$
C_{\mathcal{H}}=\sum_{i} X_{i}^{2}
$$

Hereafter the notation $C_{\mathcal{H}}$ is used for both the Casimir operator and its norm. The total variance (8) can now be represented as follows

$$
\mathbb{V}(\psi)=2\left(C_{\mathcal{H}}-\sum_{i}\left\langle\psi\left|X_{i}\right| \psi\right\rangle^{2}\right)=2\left(C_{\mathcal{H}}-R^{2}(\psi)\right) .
$$

The completely entangled states $\psi_{C E}$ obey the condition $[10,13,14]$

$$
\left\langle\psi_{C E}\left|X_{i}\right| \psi_{C E}\right\rangle=0 \quad \text { for all } X_{i} \in \operatorname{Basis}(\mathcal{L})
$$


and hence realize maximum of the total uncertainty:

$$
\mathbb{V}_{\max } \equiv \mathbb{V}\left(\psi_{C E}\right)=2 C_{\mathcal{H}} .
$$

For example, in the case of two qubits $C_{\mathcal{H}}=S(S+1)=3 / 4$, so that $\mathbb{V}_{\max }=3 / 2$ and also it is easy to check that $\mathbb{V}_{\min }=1$.

It is now seen that the concurrence (7) can be rewritten in the following way

$$
C(\psi)=\sqrt{1-\frac{R^{2}(\psi)}{R^{2}\left(\psi_{\mathrm{sep}}\right)}},
$$

where $\psi_{\text {sep }}$ denotes separable bipartite states. Since $R^{2}\left(\psi_{\text {sep }}\right)$ is a known number for any $d$ (for example, $R^{2}\left(\psi_{\mathrm{sep}}\right)=1 / 4$ for separable two-qubit states), the representation of concurrence (10) is specified by only one local parameter (4). The representation of concurrence (10) is equivalent to (7) for any $d$, which, in turn, is equivalent to (2), at least in the case of two-qubit pure states [12]. Postponing the proof of equivalence of the two representations (10) and (2) till Appendix, we concentrate now on the important case of two qubits. It is easily seen that $R^{2}\left(\psi_{\text {sep }}\right)=1 / 4$ in this case, so that Eq. (10) takes the form

$$
C(\psi)=\sqrt{1-4 R^{2}(\psi)} .
$$

The amount of specific entanglement information carried by quantum correlations between the parties in a two-qubit system is given by the entanglement of formation [4]

$$
\mathcal{E}(\psi)=h\left(\frac{1-\sqrt{1-C^{2}(\psi)}}{2}\right),
$$

where

$$
h(t) \equiv-t \log _{2} t-(1-t) \log _{2}(1-t) .
$$

It is now seen that this quantity (11) is simply expressed in terms of the local quantity $R(\psi)$ as follows

$$
\mathcal{E}(\psi)=h\left(\frac{1}{2}-R(\psi)\right)=h\left[R\left(\psi_{\mathrm{sep}}\right)-R(\psi)\right] .
$$

In other words, the amount of entanglement information is determined by deviation of the length of vector $R(\psi)$ from its value in the case of separable states. This equation (12) also shows that the local quantity $R(\psi)$ itself represents a measure of entanglement. In the case of two qubits under consideration, this quantity is defined by Eq. (5) and hence has a simple physical meaning of sum of squared mean values of spin-projection operators. An important application for the case of two qubits is provided by the polarization of photon twins (biphotons) that are created by the 
type-II down-conversion [15]. The spin operators $S_{j}$ can be associated with the Stokes operators

$$
\begin{aligned}
S_{x} & \sim\left(a_{H}^{+} a_{V}+a_{V}^{+} a_{H}\right) / \sqrt{2}, \\
S_{y} & \sim i\left(a_{H}^{+} a_{V}-a_{V}^{+} a_{H}\right) / \sqrt{2}, \\
S_{z} & \sim a_{H}^{+} a_{H}-a_{V}^{+} a_{V},
\end{aligned}
$$

so that the measurement of either concurrence (10) or entanglement of formation (12) assumes measurement of three Stokes operators for either outgoing photon beam. Here $a_{H}\left(a_{V}\right)$ denotes the photon annihilation operator with horizontal (vertical) polarization. The polarization of photons is known to be measured by means of either standard six-state or a minimal four-state ellipsometer [16].

Nevertheless, there is a certain problem with simultaneous measurement of polarization for one of the two photons created at once and forming an entangled couple. Because of the commutation relation

$$
\left[S_{j}, S_{k}\right]=i \epsilon_{j k m} S_{m}, \quad j, k, m=x, y, z,
$$

the three projections of spin (or three Stokes operators) cannot be measured independently. The minimal uncertainty relation by Schrödinger [17] states

$$
V\left(\psi ; S_{j}\right) V\left(\psi ; S_{k}\right)-\left(\operatorname{Cov}\left(S_{j}, S_{k}\right)\right)^{2} \geq \frac{1}{4}\left|\left\langle\psi\left|\left[S_{j}, S_{k}\right]\right| \psi\right\rangle\right|^{2},
$$

where $V\left(\psi ; S_{j}\right)$ denotes variance (uncertainty) of observable $S_{j}$ in the state $\psi$ and covariance $\operatorname{Cov}\left(S_{j}, S_{k}\right)$ has the form

$$
\operatorname{Cov}\left(S_{j}, S_{k}\right)=\frac{1}{2}\left\langle\psi\left|S_{j} S_{k}+S_{k} S_{j}\right| \psi\right\rangle-\left\langle\psi\left|S_{j}\right| \psi\right\rangle\left\langle\psi\left|S_{k}\right| \psi\right\rangle
$$

It is straightforward matter to see that the uncertainty relation is simply reduced to the following one

$$
0 \leq R(\psi) \leq 1 / 2
$$

where $R(\psi)$ is defined by Eq. (5). Thus, the uncertainty relation (14) becomes an exact equality when $\psi=\psi_{\text {sep }}$ with $R\left(\psi_{\text {sep }}\right)=1 / 2$. In other words, this is an unentangled biphoton state in which each photon has well-defined polarization.

In the case of completely entangled biphoton state, the quantity (5) has zero value (due to the condition (9)). In this case, the measurement performed on a single photon rises an additional question: how to distinguish between entanglement and classical unpolarized state.

Since Eq. (15) is the only relation, connecting different components of the average spin vector in either party, the local quantity (5) cannot be detected by either single or even two measurements.

Summarizing, we have shown that concurrence (2) can be expressed in terms of only local quantity (4), measured for either party of the system. In other words, 
any party of a composite system carries complete information about the amount of entanglement caused by quantum correlations between the parties. The rigorous proof of equivalence of representations (2) and (10) for an arbitrary $d$ is given in Appendix.

The preponderance of the measure (4) is that it expresses the amount of entanglement in an operational way via averages of physical observables rather than in terms of complex coefficients of the state (1).

According to our result, the concurrence is characterized by deviation of the local quantity $R(\psi)$ from its value measured for a separable state. This deviation is associated with increase of total uncertainty in the system with respect to "classical" level given by unentangled states. Representations of concurrence (10) and (7) reflect the physical nature of entanglement as manifestation of quantum uncertainty above the "classical" level provided by the separable states (for discussion of entanglement in the context of quantum uncertainties, see Ref. [10] and references therein).

Generally speaking, detection of the quantity (4) assumes measurement of all averages $\left\langle\psi\left|X_{i}\right| \psi\right\rangle$. The practical realization of those measurement even in the simplest case of two qubits requires special consideration.

We have considered measure of entanglement of only bipartite pure states. The possible extension of the result to the case of multipartite pure states and bipartite mixed states seems to be an interesting problem for further discussion.

\section{Appendix}

Our aim is now to prove that the representations (2) and (10) or (7) give the same amount of entanglement for pure states of a bipartite system of an arbitrary dimension $d$. It seems to be convenient to prove validity of these two representations in general settings without specification of parties of the system.

It is seen from Eq. (10) that operational representation of concurrence depends on the state $\psi$ only via quadratic form $R^{2}(\psi)$ given by Eq. (4).

Consider the space $\operatorname{Herm}(\mathcal{H})$ of all Hermitian operators in $\mathcal{H}$ with trace metric $\operatorname{Tr}_{\mathcal{H}}(X Y)$. Let $\rho_{\mathcal{L}}$ be the orthogonal projection of $\rho \in \operatorname{Herm}(\mathcal{H})$ onto subalgebra $\mathcal{L} \subset \operatorname{Herm}(\mathcal{H})$, so that $\operatorname{Tr}_{\mathcal{H}}(\rho X)=\operatorname{Tr}_{\mathcal{H}}\left(\rho_{\mathcal{L}} X\right)$. We can now choose $\rho_{\mathcal{L}} / \sqrt{\left(\rho_{\mathcal{L}}, \rho_{\mathcal{L}}\right)_{K}} \in \mathcal{L}$ as one of the unit base vectors $X_{i} \in \mathcal{L}$ (a basic observable). The remaining base vectors are orthogonal to $\rho_{\mathcal{L}}$, so that

$$
R^{2}(\rho)=\sum_{i}\left[\operatorname{Tr}\left(\rho_{\mathcal{L}} X_{i}\right)\right]^{2}=\frac{\operatorname{Tr}_{\mathcal{H}}\left(\rho_{\mathcal{L}}^{2}\right)^{2}}{\left(\rho_{\mathcal{L}}, \rho_{\mathcal{L}}\right)_{K}}
$$

The invariant trace form which appears here is proportional to the Killing form $(X, Y)_{K}$ of $\mathcal{L}$

$$
\operatorname{Tr}_{\mathcal{H}}(X Y)=D_{\mathcal{H}} \cdot(X, Y)_{K}, \quad X, Y \in \mathcal{L}
$$

with coefficient $D_{\mathcal{H}}$ known as the Dynkin index (concerning Killing form and Dynkin 
index, see Ref. [18]). Now, the total uncertainty (8) can be represented as follows

$$
\mathbb{V}(\psi)=C_{\mathcal{H}}-D_{\mathcal{H}}\left(\rho_{\mathcal{L}}, \rho_{\mathcal{L}}\right)_{K},
$$

where $C_{\mathcal{H}}$ again denotes the Casimir operator. For the Lie algebra $\mathcal{L}$ under consideration, the coefficients $C_{\mathcal{H}}$ and $D_{\mathcal{H}}$ are given by the following equations

$$
C_{\mathcal{H}}=(\lambda, \lambda+2 \delta), \quad D_{\mathcal{H}}=\frac{\operatorname{dim} \mathcal{H}}{\operatorname{dim} \mathcal{L}}(\lambda, \lambda+2 \delta)
$$

where $\lambda$ denotes the highest weight of irreducible representation $\mathcal{H}$ and $2 \delta$ is the sum of positive roots of $\mathcal{L}$. In general, the Lie algebra $\mathcal{L}$ splits into direct sum of simple components $\mathcal{L}=\bigoplus_{j} \mathcal{L}_{(j)}$ and every its irreducible representation $\mathcal{H}$ decomposes into the tensor product $\mathcal{H}=\bigotimes_{j} \mathcal{H}_{j}$ of irreducible representations $\mathcal{L}^{(j)}: \mathcal{H}_{j}$. Under this setting, the Casimir and Dynkin index can be found from the following additivity property

$$
C_{\mathcal{H}}=\sum_{j} C_{\mathcal{H}_{j}}, \quad \frac{D_{\mathcal{L}}}{\operatorname{dim} \mathcal{H}}=\sum_{j} \frac{D_{\mathcal{H}_{j}}}{\operatorname{dim} \mathcal{H}_{j}} .
$$

In the case of bipartite systems under consideration, index $j=A, B, \operatorname{dim} \mathcal{H}_{A}=$ $\operatorname{dim} \mathcal{H}_{B}$, and the dynamic symmetry group $G=\mathrm{SU}\left(\mathcal{H}_{A}\right) \times \mathrm{SU}\left(\mathcal{H}_{B}\right)$. The corresponding Lie algebra $\mathcal{L}=\operatorname{Lie}(G)$ consists of the operators

$$
X=X^{A} \otimes \mathbf{1}+\mathbf{1} \otimes X^{B},
$$

where 1 denotes the identity operator and $X^{A, B}$ are the traceless Hermitian operators in $\mathcal{H}_{A}$ and $\mathcal{H}_{B}$. By definition of reduced states $\rho^{A}, \rho^{B}$, we have

$$
\operatorname{Tr}_{\mathcal{H}}(\rho X)=\operatorname{Tr}_{\mathcal{H}}\left(\rho^{A} X^{A}\right)+\operatorname{Tr}_{\mathcal{H}}\left(\rho^{B} X^{B}\right),
$$

and therefore the projection $\rho_{\mathcal{L}}$ of $\rho$ into $\mathcal{L}$, characterized by equation $\operatorname{Tr}_{\mathcal{H}}(\rho X)=$ $\operatorname{Tr}_{\mathcal{H}}\left(\rho_{\mathcal{L}} X\right)$, amounts to

$$
\rho_{\mathcal{L}}=\frac{1}{n}\left(\rho_{0}^{A} \otimes \mathbf{1}+\mathbf{1} \otimes \rho_{0}^{B}\right)
$$

where the subscript 0 denotes the traceless parts of $\rho^{A, B}$. A simple calculation shows that $\operatorname{Tr}\left(\rho_{\mathcal{L}}^{2}\right)$ is a linear function in $\operatorname{Tr}\left(\left[\rho^{A}\right]^{2}\right)$ and $\operatorname{Tr}\left(\left[\rho^{B}\right]^{2}\right)$. It is also seen that for the pure state under consideration $\operatorname{Tr}\left(\left[\rho^{A}\right]^{2}\right)=\operatorname{Tr}\left(\left[\rho^{B}\right]^{2}\right) \equiv \operatorname{Tr}\left(\rho_{r}^{2}\right)$, and that in this case both the total uncertainty $\mathbb{V}(\psi)$ and square of the concurrence $(2)$ (with factor $\nu=d /(d-1)$ instead of 2) are linear functions of $\operatorname{Tr}\left(\rho_{r}^{2}\right)$. Since the two linear functions $[C(\psi)]^{2}$, given by Eq. (2) and the normalized total uncertainty

$$
\frac{\mathbb{V}(\psi)-\mathbb{V}_{\text {min }}}{\mathbb{V}_{\max }-\mathbb{V}_{\min }}=1-\frac{R^{2}(\psi)}{R^{2}\left(\psi_{\text {sep }}\right)}
$$

have equal values for separable (unentangled) and completely entangled states, they are identical. Q.I.D. 


\section{Acknowledgments}

One of the authors (B. Ö.) would like to acknowledge the Scientific and Technical Research Council of Turkey (TÜBİTAK) for financial support.

\section{References}

1. L. Marcikic, H. de Riedmatten, W. Tittel and N. Gisin, Nature (London) 42 (2003) 509.

2. A. Poppe, A. Fedrizzi, R. Ursin, H.R. Böhm, T. Lörunser, O. Maurhardt, M. Peev, M. Suda, C. Kurtsiefer, H. Weinfurter, T. Jennewein and

A. Zeilinger, Optics Express 12 (2004) 3865.

3. J. Ouelette, The Industrial Phys. 10 (2004) 22.

4. S. Hill and W.K. Wootters, Phys. Rev. Lett. 78 (1997) 5022; W.K. Wootters, Phys. Rev. Lett. 80 (1998) 2245.

5. P. Rungta, V. Bužek, C.M. Caves, M. Hillery and G.J. Milburn, Phys. Rev. A 64 (2001) 042315.

6. F. Mintert, M. Kuś and A. Buchleitner, Phys. Rev. Lett. 92 (2004) 167902.

7. J.S. Bell, Rev. Mod. Phys. 38 (1966) 447.

8. R. Hermann, Lie Groups for physicists, Benjamin, New York, 1966.

9. A.A. Klyachko, quant-ph/0206012.

10. A.A. Klyachko and A.S. Shumovsky, J. Phys: Conf. Series 36 (2006) 87.

11. C.M. Caves and G.J. Milburn, Opt. Commun. 179 (2000) 439.

12. A.A. Klyachko, B. Öztop and A.S. Shumovsky, Appl. Phys. Lett. 88 (2006) 124102.

13. M.A. Can, A.A. Klyachko, and A.S. Shumovsky, Phys. Rev. A 66 (2002) 022111.

14. A.A. Klyachko and A.S. Shumovsky, J. Opt. B: Quant. and Semiclas. Optics 6 (2004) S29.

15. A.F. Abouraddy, B.E.A. Saleh, A.V. Sergienko and M.C. Teich, Phys. Rev. A 64 (2001) 050101(R); M.B. Nasr, A.F. Abouraddy, M.C. Booth, B.E.A. Saleh, A.V. Sergienko, M.C. Teich, M. Kempe and R. Wolleschensky, Phys. Rev. A 65 (2002) 023816; G. DiGiuseppe, M. Atature, M.D. Shaw, A.V. Sergienko, B.E.A. Saleh, M.C. Teich, A.J. Miller, S.W. Nam and J. Martinis, Phys. Rev. A 68 (2003) 063817; Yu.I. Bogdanov, M.V. Chekhova, S.P. Kulik, G.A. Maslennikov, A.A. Zhukov, C.H. Oh and M.K. Tey, Phys. Rev. Lett. 93 (2004) 230503.

16. J. Řeháček, B.G. Englert and D. Kaszlikowski, Phys. Rev. A 70 (2004) 052321.

17. E. Schrödinger, Proc. Prussian Acad. Sci. 19 (1930) 296; V.V. Dodonov, E.V. Kurmushev and V.I. Man'ko, Phys. Lett. A 79 (1980) 150;

E.C.G. Sudarshan, C.B. Chiu and G. Bhamathi, Phys. Rev. A 52 (1995) 43.

18. A.L. Onishchik and E.B. Vinberg, eds., Lie groups and Lie algebras III, Encyclopedia of Math. Science Vol. 41, Springer, Berlin, 1994. 